STRUCTURAL SCIENCE CRYSTAL ENGINEERING MATERIALS

ISSN 2052-5206

\section{Combining forces: complementary techniques brought together to determine tricky crystal structures}

\author{
Graeme M. Day* \\ School of Chemistry, University of Southampton, Southampton, S017 1NX, United Kingdom. *Correspondence e-mail: \\ g.m.day@soton.ac.uk
}

In this issue of Acta Crystallographica Section B, Dudek et al (2020) report the determination of two crystal structures of the pharmaceutical molecule furazidin, whose existence has been reported in patents, but whose structures have to date been uncharacterized. The work shows how multiple techniques - powder X-ray diffraction, nuclear magnetic resonance (NMR) spectroscopy and computational crystal structure prediction (CSP) - are sometimes required to determine crystal structures of organic molecules when direct determination from diffraction data is not possible, and demonstrates the complementarity of information provided by the different methods.

Crystallization of furazidin in this work produced microcrystalline powders and no single crystals that could be used for crystal structure determination. This is a common problem that can frustrate attempts to understand the structure of a material. However, developments in applying NMR techniques to solids, along with quantum mechanical methods that can provide accurate predicted NMR parameters from putative structures, can offer an alternative to diffraction-based structure solution. This approach of determining a crystal structure by constructing or identifying a structural model that reproduces the observed NMR spectra is termed NMR crystallography. A challenging stage in applying NMR crystallography is the generation of realistic structural models to which quantum mechanical predictions of NMR parameters can be applied.

This is where CSP has entered the field of NMR crystallography; computational methods for CSP have been developed to predict, from nothing more than the chemical diagram, the likely crystal packings of a molecule. While CSP is often discussed as an $a b$ initio method, requiring no input of experimental data, these methods can also be combined with experiment, providing the missing ingredient in NMR crystallography determination of structures. The process seems simple: apply CSP to predict possible crystal structures, perform calculations to predict the NMR parameters for the best predicted structures and compare these with the measured NMR spectra. However, CSP for molecules of the size and flexibility of furazidin is challenging, particularly when the experimental information shows that there are two symmetrically unrelated molecules in one of the polymorphs. CSP is relied on in this method to locate all possible structures, which correspond to local energy minima on the energy surface defined by all degrees of freedom of the crystal structure. Molecular flexibility and multiple independent molecules add to these degrees of freedom and lead to very large numbers of energy minima, often numbering in the hundreds or thousands within the usual energetic range of polymorphism. This creates a computational challenge in itself - the location of all possible structures - and creates a second problem for NMR crystallography: within a large set of computer-generated crystal structures, one can find false-positive agreement with the measured NMR spectra. These are structures where calculated chemical shifts seem to be close enough to experiment (within the errors of the quantum mechanical methods used to predict the NMR), but where this agreement is fortuitous, and the structure is incorrect.

The complexity of CSP is reduced in this work by identifying constraints from the experimental data to the degrees of freedom that need to be explored. Diffraction data and NMR are able to provide complementary views of the structure; NMR gives local structural information, reporting on molecular geometry, close contacts and nearest 


\section{scientific commentaries}

neighbour interactions, while the most readily extracted information from diffraction is the long-range periodicity of the molecular arrangement. Powder X-ray diffraction was used to narrow the list of possible space-group symmetries that needed to be considered during structure prediction, while NMR provided information to narrow the set of molecular conformations, which further reduced the search space. This led to a tractable CSP problem.

The issue of false positives was addressed by comparing calculated and measured NMR parameters for multiple nuclei - here, both ${ }^{1} \mathrm{H}$ and ${ }^{13} \mathrm{C}$. While incorrect structures sometimes reproduced either the measured ${ }^{1} \mathrm{H}$ or ${ }^{13} \mathrm{C}$ chemical shifts, only one structure for each polymorph was found to reproduce both. This in an important message from this study: not to rely solely on ${ }^{1} \mathrm{H}$ or ${ }^{13} \mathrm{C}$ data because of the risk that agreement with experiment is spurious.

The report by Dudek and coworkers of integrating these techniques - diffraction, NMR and structure prediction - to determine crystal structures that could not have been determined using a single method is an important example that nicely illustrates the process and demonstrates the importance of careful validation.

\section{References}

Dudek, M. K., Paluch, P. \& Pindelska, E. (2020). Acta Cryst. B76, 322335. 